\title{
Cryptographic key assignment scheme for dynamic access control in a user hierarchy
}

\author{
F.H.Kuo, V.R.L.Shen, T.S.Chen and F.Lai
}

\begin{abstract}
The authors present a cryptographic key assignment scheme based on both the Rabin public key system and the Chinese remainder theorem, in order to solve dynamic access control problems in user hierarchies. Compared to earlier assignment schemes, the new scheme ensures that a security class can efficiently derive secret keys of its successors. Dynamic access control problems, such as adding/deleting classes, adding/deleting relationships, and changing secret keys, are considered. Moreover, without altering other keys present in the system, each user in the hierarchy can freely change his own key.
\end{abstract}

\section{Introduction}

In computer communication systems, both computer cryptography and information security have become important requirements. The unauthorised user is not allowed to access secret data, and such data must be well protected. Due to the growth of computer networks, and the fast progress of computer technologies on multi-user systems, sharing resources has become common, and the administration of these resources is important in a multi-user computer environment. In other words, access control through authorisation becomes important.

The access control problem in a computer environment can be described by a user hierarchy. In such a hierarchy, the users and their own information items are organised into a number of disjoint sets of security classes, and each user is assigned to a security class called the user's 'security clearance'. Let $S C_{1}, S C_{2}, \ldots, S C_{n}$ be $n$ disjoint security classes. Assume that " $\leq$ " is a binary partially ordered relation on the set $S C=\left\{S C_{1}, S C_{2}, \ldots, S C_{n}\right\}$. Then, in the partially ordered set (poset) $(S C, \leq), S C_{j} \leq S C_{i}$ means that the users in the security class $S C_{i}$ have a security clearance higher than or equal to those in the security class $S C_{j}$. In other words, users in the security class $S C_{i}$ can read and store information items that belong to users in the security class $S C_{j}$. In contrast, the users in $S C_{j}$ cannot read or store information items in $S C_{i}$.

\section{(C) IEE, 1999}

IEE Proceedings online no. 19990311

DOI: 10.1049/ip-cdt:19990311

Paper received 4th February 1999

F.-H. Kuo is with the Department of Electrical Engineering, National Taiwan University, Taipei 106, Taiwan

V.R.L. Shen is with the Department of Electrical Engineering, Mingchi Institute of Technology, P.O. Box 3-28, Hsinchuang City, Taipei 243, Taiwan

E-mail: rlshen@ccson.mit.edu.tw

T.S. Chen is with the Department of Computer Science and Information Engineering, Da Yeh University

F. Lai is with the Department of Electrical Engineering and Department of Computer Science and Information Engineering, National Taiwan University, Taipei 106, Taiwan

IEE Proc.-Computers \& Digital Techniques, Vol. 146, No. 5, September 1999
Fig. 1 shows an example of a poset connected to a user hierarchy. The arrowhead in Fig. 1 indicates that the higher level security classes have a security clearance higher than the lower level ones. $S C_{j} \leq S C_{i}$ means that $S C_{i}$ is the predecessor of $S C_{j}$; and $S C_{j}$ a successor of $S C_{i}$. Furthermore, if there is no security class $S C_{k}$ in $S C$ such that $S C_{j} \leq S C_{k} \leq S C_{i}$, then $S C_{i}$ is called an immediate predecessor of $S C_{j}$ and $S C_{j}$ an immediate successor of $S C_{i}$.

In many application areas there is a need to carry out classified data management, so access control problems become very important. Access control in a hierarchy can be used not only in military and government departments but also in private corporations. Key assignment schemes are for instance applicable in database management systems [1-3], computer networks [4, 5] and operating systems [6].

The main problem is how the user in a security class can derive the secret key for users in a lower security class. Obviously, the easiest way to accomplish this is that the users keep all the keys of their successors in a database. As shown in Fig. 1, if the user in $S C_{1}$ wants to retrieve the data which are encrypted under his own key or his successors' keys, he needs a set of secret keys $\left\{S K_{1}, S K_{2}, S K_{3}, S K_{4}\right.$, $\left.S K_{5}, S K_{6}\right\}$. However, when the hierarchy becomes quite large, the users with the higher privileges need a great number of secret keys, and the quantity of keys thus becomes inconvenient to administer and hazardous to keep secure. Therefore, our goal is to find a method in which each user only needs to hold one key to retrieve all information which he is entitled to access, and to still retain the security of the system.

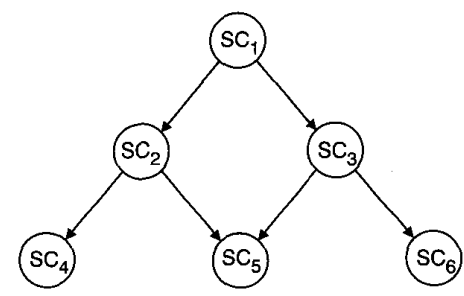

Fig. 1 The poset in a user hierarchy 
When a user in security class $S C_{i}$ wants to store the plaintext $M$ into the database or broadcast it to the network, he first uses his secret key $S K_{i}$ to encrypt $M$, then he will store or broadcast the ciphertext $C=E_{S K i}(M)$. Only the users with $S K_{i}$ can get $M$ by calculating $M=D_{S K i}(C)$, where $E$ and $D$ represent the prepublished encryption and decryption algorithms, respectively. For $S C_{j} \leq S C_{i}, S C_{i}$ can adopt his secret key $S K_{i}$ and $S C_{j}$ 's public information to derive $S C_{j}$ 's secret key $S K_{j}$, and then reads the information items possessed by $S C_{j}$.

Our scheme is composed of Rabin's cryptosystem and the Chinese remainder theorem (CRT). Rabin's cryptosystem is provably as secure as factoring [7], and the CRT has been shown to increase the deciphering speed in RSA public-key cryptosystem fourfold [8]. This motivates us to propose the novel scheme which has the following properties.

1. Key generation and key derivation algorithms are simple.

2. Dynamic access control problems, such as adding/ deleting classes, adding/deleting relationships, and changing secret keys, can be overcome easily.

3 . Users can change their secret keys easily.

4. The system can withstand the attack of collusion.

\section{Review of previous research}

Under the property that $t_{i} \mid t_{j}$ (i.e. $t_{j}$ is divisible by $t_{i}$ ) if and only if $S C_{j} \leq S C_{i}$, Akl and Taylor [9] first proposed that each security class $S C_{i}$ was assigned a public integer $t_{i}$. Then the secret key $S K_{i}$ of $S C_{i}$ is computed according to $S K_{i}=S K_{0}^{t i}(\bmod m)$, where $S K_{0}$ is the secret key of central authority (CA) and $m$ is a product of a secret pair of large prime numbers. If $S C_{j} \leq S C_{i}, t_{j} / t_{i}$ is an integer and $S C_{i}$ can derive $S K_{j}$ by the deduction:

$$
S K_{j}=S K_{0}^{t j}=S K_{0}^{t^{i *}(t j / t i)}=S K_{i}^{(t j / t i)}(\bmod m) .
$$

On the contrary, if $S C_{j}$ (not $\leq$ ) $S C_{i}$, then $t_{j} / t_{i}$ is not an integer and the key derivation will fail. However, the size of the public information $t_{i}$ will increase dramatically when the number of security classes becomes large.

The advantage of Akl and Taylor's scheme is that the key generation and key derivation algorithms are quite simple; however, several drawbacks exist. First, when the number of security classes in the hierarchy is large, a large amount of storage is required for the public parameters. Secondly, as to the dynamic access control problems, such as adding/ deleting classes or relationships in the existing system, the whole system has to be reestablished. Third, it is rather difficult to change the secret key.

Hence, MacKinnon et al proposed an improved method to reduce the values of $t_{i}$ by using a canonical assignment method [10]. Later, Harn et al presented another method which uses a bottom-up key generation scheme to replace the top-down one $[9,11]$. As a result, the values of $t_{i}$ indeed become smaller than those in the Akl-Taylor scheme.

Whenever a new security class is added into the user hierarchy system, the schemes mentioned above cannot satisfy the security requirements. Unless the issued keys are changed, it is impossible to add a security class to the system.

Therefore, several key management schemes are proposed to solve the dynamical adding or deleting of the security class, and to keep the size of public information items as small as possible [12-15]. In these schemes, the user with higher security clearance must iteratively perform the key derivation process for deriving the secret key of the user who is not the immediate successor. And the key derivation is inefficient. Consequently, some other schemes, proposed to extend the Akl-Taylor scheme, allow the users to change their keys and to derive any secret keys belonging to the successors efficiently $[16,17]$.

Meanwhile, each aforementioned scheme allows any security class to derive the keys of his successors from his own secret key, and to prevent the possibility of collaboration by the successors to derive his own secret key.

\section{Proposal for a new scheme}

Based on Rabin's public key system and the CRT, our scheme is intended to solve dynamic access control problems in user hierarchies.

\subsection{Rabin's public key system}

In Rabin's public key system [18], there are a pair of public keys $(b, m)$ for encryption, and a pair of secret keys $(p, q)$ and $b$ for decryption. The key point for Rabin's cryptosystem is to select a pair of secret and large prime numbers $(p, q)$ and to let $m=p * q$. The encryption procedure $E$ is shown as follows:

$$
C=E(M)=M(M+b) \bmod m,
$$

where $M$ is a plaintext, and $C$ is a ciphertext. The decryption procedure $D$ is to obtain solutions for $M$ through the following congruence:

$$
M^{2}+M b-C=0 \bmod m .
$$

Since the module $m$ is equal to $p * q$, eqn. 2 will be equivalent to the following two congruences:

$$
\begin{aligned}
& M^{2}+M b-C=0 \bmod p, \\
& M^{2}+M b-C=0 \bmod q .
\end{aligned}
$$

Since eqns. 3 and 4 are quadratic congruences, the plaintext $M$ can thus be recovered through the following four square roots modulo $p q$ :

$$
\begin{aligned}
& M=\frac{-b}{2}+\sqrt{\left(\frac{-b}{2}\right)^{2}+C} \bmod p, \\
& M=\frac{-b}{2}-\sqrt{\left(\frac{-b}{2}\right)^{2}+C} \bmod p, \\
& M=\frac{-b}{2}+\sqrt{\left(\frac{-b}{2}\right)^{2}+C} \bmod q, \\
& M=\frac{-b}{2}-\sqrt{\left(\frac{-b}{2}\right)^{2}+C} \bmod q .
\end{aligned}
$$

Obviously, Rabin's system is popular in the public key cryptosystems. The encryption function of the system is based on a second-order polynomial, which computes the modulus of the product of two large primes $(p, q)$; and the decryption function on a square-root function, which computes the modulus of one of these two primes. Thus, Rabin's system makes our scheme elegant and practical in computation.

If a user wants to derive the secret key $S K$, first he must own the secret data $p$ and $q$ of the decryption procedure $D$. In other words, the security of the system, based on the 
difficult computation of the factoring of the product of two large primes, will be broken when one pair of primes $(p, q)$ exists. Therefore, in order to keep the security of the system, we need to give each security class a distinct pair of primes. Dirichlet's theorem [19] thus offers the distinct pair of primes as follows:

Let $b \geq 1$, then there will exist infinite integers $r \geq 1$ such that $r * 2^{b}+1$ is one prime, and also exist infinite integers $r^{\prime} \geq 1$ such that $r^{\prime} * 2^{b}-1$ is the other prime.

In order to generate its own secret primes $p$ and $q$ under the security of the system, each security class should hold two secret information items $\left(r, r^{\prime}\right)$ and a public datum $b$. In other words, when a user $U_{i}$ with a higher security clearance wants to decrypt the information items held by his successor $U_{j}$, the secret data $\left(r_{j}, r_{j}\right)$ of $U_{j}$ should first be obtained; then $p_{j}$ and $q_{j}$, which perform the decryption function, can be derived. As for $U_{i}$, holding all his successors' information items $\left(r, r^{\prime}\right)$ is as awkward and insecure as holding all his successors' keys. Consequently, we include the CRT to overcome this difficulty.

\subsection{Chinese remainder theorem (CRT)}

Let $n_{1}, n_{2}, \ldots, n_{t}$ be pairwise relative primes, i.e. $\operatorname{gcd}\left(n_{i}\right.$, $\left.n_{j}\right)=1$, for $i \neq j$. Let $r_{1}, r_{2}, \ldots, r_{i}$ be integers. Then the congruent equation is of the form: $H \equiv r_{i}\left(\bmod n_{i}\right)$.

Let $N=n_{1} n_{2} \ldots n_{t}$. There is only a unique solution $H$ between 0 and $N-1$. The solution of $H$ is of the form:

$$
H=\sum_{i=1}^{t} r_{i} X_{i}(\bmod N)
$$

where $X_{i}=y_{i} *\left(N / n_{i}\right)$ and $y_{i}$ satisfies $y_{i} *\left(N / n_{i}\right) \equiv 1\left(\bmod n_{i}\right)$.

\subsection{Proposed scheme}

In order to find the true plaintext, we propose adding some information into the original plaintext $M$, such as sender identity code, receiver identity code, date, and time. In our scheme, the secret key $S K$ concatenated with the specific identity code (ID) is used to replace the message $M$ in the above encryption procedure $E$, so eqn. 1 is modified to

$$
C=(S K \| I D)((S K \| I D)+b) \bmod m,
$$

where " $\|$ " represents the concatenation operation. Undoubtedly, the secret key $S K$ can be recovered correctly. Also, a 4:1 ambiguity in the decrypted messages [7] can be avoided in this manner.

3.3.1 Key generation algorithm. We suppose that the existing system has $n$ security classes $S C_{1}, S C_{2}, \ldots$, and $S C_{n}$ in the user hierarchy. The algorithm of generating the secret key $S K_{i}$ for each security class $S C_{i}$ is stated as follows:

Step 1: Randomly select $n$ coprime integers $n_{1}$, $n_{2}, \ldots, n_{n}$ to be as small as possible.

Step 2: Compute $N=\Pi_{i=1}^{n} n_{i}$.

Step 3: Take a node $S C_{i}$ from the hierarchy by preorder traversal.

Step 4: CA (i.e. Central Authority) randomly selects three positive integers $r_{i}, r_{i}^{\prime}$ and $b_{i}$ for security class $S C_{i}$ such that $\left(r_{i} * 2^{b i}+1\right)$ and $\left(r_{i}^{\prime} * 2^{b i}-1\right)$ are equal to prime numbers $p_{i}$ and $q_{i}$, respectively.

Step 5: CA chooses $y_{i}$ such that $\left(N / n_{i}\right) * y_{i} \bmod n_{i}=1$ for $1 \leq i \leq n$, and lets $X_{i}=\left(N / n_{i}\right) * y_{i}$. CA then computes each security class $S C_{i}^{\prime}$ s derivation information

$$
H_{i}=\Sigma_{j}\left(r_{j} \| r_{j}^{\prime}\right) * X_{j}-\left(r_{i} \| r_{i}^{\prime}\right) * X_{i}(\bmod N) \text { for } S C_{j} \leq S C_{i}
$$

Step 6: When the security class $S C_{i}$ receives the data of $\left(r_{i}, r_{i}^{\prime}, X_{i}, H_{i}\right)$ and its own public parameters $\left(b_{i}, n_{i}\right), \mathrm{CA}$ performs the following steps:

6.1 Compute $p_{i}=\left(r_{i} * 2^{b i}+1\right), q_{i}=\left(r_{i}^{\prime} * 2^{b i}-1\right)$, let $m_{i}=p_{i} * q_{i}$, and keep $p_{i}, q_{i}$, and $m_{i}$ secret.

6.2 Choose one secret key $S K_{i}$ for convenience, then concatenate the secret key $S K_{i}$ with its identity code $I D_{i}$, and let its range be in $\left[1, m_{i}\right]$, In other words, let $M_{i}$ be equal to $S K_{i}$ concatenated with $I D_{i}$, i.e. $M_{i}=S K_{i} \| I D_{i}$, where $1 \leq M_{i} \leq m_{i}-1$.

6.3 Calculate the public information $C_{i}=\left(M_{i}\right) *$ $\left(M_{i}+b_{i}\right) \bmod m_{i}$.

Step 7: Repeat steps 3 to 7 until all nodes of the hierarchy are traversed.

3.3.2 Key derivation algorithm. By the $S C_{i}$ successors' public parameters, $S C_{i}$ can derive any successor $S C_{j}^{\prime} \mathrm{s}$ secret key $S K_{j}$, then, by $S K_{j}, S C_{i}$ decrypts the information items possessed by $S C_{j}$. The algorithm to derive the secret key $S K_{j}$ is stated as follows:

Step 1: Compute $\left(r_{j} \| r_{j}^{\prime}\right)=H_{i} \bmod n_{j}$.

Step 2: Compute $p_{j}=r_{j} * 2^{b j}+1 ; q_{j}=r_{j}^{\prime} * 2^{b j}-1$.

Step 3: Due to the same reasons as in Section 3.1, compute

$$
\begin{aligned}
& M_{j}=\frac{-b_{j}}{2}+\sqrt{\left(\frac{-b_{j}}{2}\right)^{2}+C_{j}} \bmod p_{j}, \\
& M_{j}=\frac{-b_{j}}{2}-\sqrt{\left(\frac{-b_{j}}{2}\right)^{2}+C_{j}} \bmod p_{j}, \\
& M_{j}=\frac{-b_{j}}{2}+\sqrt{\left(\frac{-b_{j}}{2}\right)^{2}+C_{j}} \bmod q_{j}, \\
& M_{j}=\frac{-b_{j}}{2}-\sqrt{\left(\frac{-b_{j}}{2}\right)^{2}+C_{j}} \bmod q_{j} .
\end{aligned}
$$

Step 4: According to the identity code $I D_{j}$, extract $S K_{j}$ from the true plaintext $M_{j}$.

\section{Dynamic access control}

This section presents the dynamic access control problems, including adding/deleting classes, adding/deleting relationships, and changing secret keys.

\subsection{Adding classes}

To add a new security class $S C_{n+1}$ into an existing hierarchy, CA must update the $H_{i}$ of those security classes $S C_{i}$ which serve as predecessors. All other keys in the system can stay the same. Suppose that a new security class $S C_{n+1}$ is added to the existing system. CA performs the following steps:

Step 1: Select a new prime number $n_{n+1}$

Step 2: $\quad N=N * n_{n+1}$.

Step 3: Calculate $y_{n+1}$ such that $\left(N / n_{n+1}\right) * y_{n+1} \bmod$ $n_{n+1}=1$, and let $X_{n+1}=\left(N / n_{n+1}\right) * y_{n+1}$.

Step 4: Randomly select three positive integers $r_{n+1}$, 
$r_{n+1}^{\prime}$ and $b_{n+1}$ such that

$$
\begin{aligned}
& p_{n+1}=\left(r_{n+1} * 2^{b n+1}+1\right), \\
& q_{n+1}=\left(r_{n+1}^{\prime} * 2^{b n+1}-1\right), \\
& \text { and let } m_{n+1}=p_{n+1} * q_{n+1} .
\end{aligned}
$$

Step 5: For all the predecessors $S C_{i}$ of $S C_{n+1}$, after CA adds the new security class $S C_{n+1}$

$$
H_{i}=H_{i}+\left(r_{n+1} \| r_{n+1}^{\prime}\right) * X_{n+1}(\bmod N) .
$$

Step 6: Derive $H_{n+1}=\Sigma_{j \in J}\left(r_{j} \| r_{j}^{\prime}\right) * X_{j}-\left(r_{n+1} \| r_{n+1}^{\prime}\right) *$ $X_{n+1}(\bmod N)$, where $\boldsymbol{J}=\left\{j \mid\left(S C_{j} \leq S C_{n+1}\right)\right.$ AND NOT $\left.\left(S C_{j} \leq S C_{i}\right)\right\}$.

Step 7: Compute $C_{n+1}=\left(M_{n+1}\right) *\left(M_{n+1}+b_{n+1}\right) \quad(\bmod$ $\left.m_{n+1}\right)$, where $M_{n+1}=S K_{n+1} \| I D_{n+1}$.

Example 1: Let $S C_{7}$ be a new security class which is added as a successor of $S C_{1}$, as shown in Fig. 2. Since only $S C_{1}$ has the access right to the new class $S C_{7}$, CA needs to reconstruct $H_{1}$ of the security class $S C_{1}$ after the secret information $X_{7}$ of $S C_{7}$ has been generated. CA will select these parameters $\left(n_{7}, y_{7}, r_{7}, r_{7}^{\prime}, b_{7}\right)$ and recompute the following equations.

$$
\begin{aligned}
N & =N * n_{7}, X_{7}=\left(N / n_{7}\right) * y_{7} \\
p_{7} & =\left(r_{7} * 2^{b 7}+1\right), q_{7}=\left(r_{7}^{\prime} * 2^{b 7}-1\right), m_{7}=p_{7} * q_{7} \\
H_{1} & =H_{1}+\left(r_{7} \| r_{7}^{\prime}\right) * X_{7}(\bmod N) \\
H_{7} & =\left[\left(r_{7} \| r_{7}^{\prime}\right) * X_{7}+\left(r_{6} \| r_{6}^{\prime}\right) * X_{6}-\left(r_{7} \| r_{7}^{\prime}\right) * X_{7}\right](\bmod N) \\
C_{7} & =\left(S K_{7} \| I D_{7}\right) *\left(\left(S K_{7} \| I D_{7}\right)+b_{7}\right)\left(\bmod m_{7}\right)
\end{aligned}
$$

\subsection{Deleting classes}

$\mathrm{CA}$ will perform the following steps to delete a security class $S C_{k}$ in the existing system. For all the predecessors $S C_{i}$ of $S C_{k}$, CA needs to have to recompute $N$ and $H_{i}$. Then, $\mathrm{CA}$ will discard the secret data and public parameters of $S C_{k}$.

Step 1: $\quad N=N / n_{k}$.

Step 2: For all the predecessor $S C_{i}$ of $S C_{k}$, before CA deletes the $S C_{k}$,

$$
H_{i}=H_{i}-\left(r_{k} \| r_{k}^{\prime}\right) * X_{k}(\bmod N) .
$$

Step 3: Discard the secret data and public parameters of $S C_{k}$.

Example 2: Suppose that $S C_{3}$ in Fig. 1 is deleted from the hierarchy; the resulting hierarchy is shown in Fig. 3. $\mathrm{CA}$ will recompute the following equations.

$$
N=N / n_{3}, H_{1}=H_{1}-\left(r_{3} \| r_{3}^{\prime}\right) * X_{3}(\bmod N)
$$

Then, CA will discard the secret data $\left(r_{3}, r_{3}^{\prime}, X_{3}, H_{3}\right)$ and the public parameters $\left(b_{3}, n_{3}\right)$.

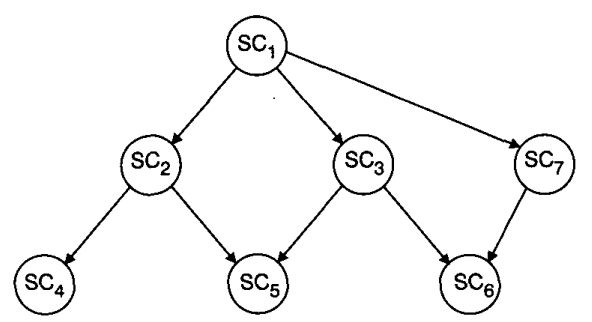

Fig. 2 Adding a new security class $S C_{7}$

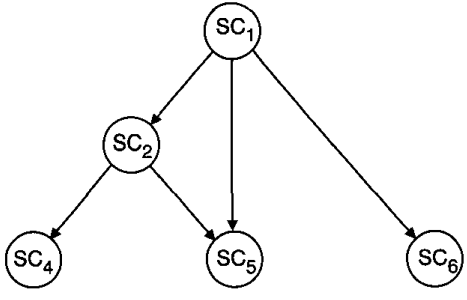

Fig. 3 Deleting the security class $\mathrm{SC}_{3}$

\subsection{Adding a relationship}

Suppose that a new relationship between $S C_{b}$ and $S C_{a}$ is added into a user hierarchy such that $S C_{b} \leq S C_{a}$. CA only recomputes the public parameters $H_{i}$ s for $S C_{b} \leq S C_{i}$, while other parameters remain unchanged. Then, after $\mathrm{CA}$ adds the relationship of $S C_{b} \leq S C_{a}$, it will perform the following procedure:

Step 1: For $S C_{b} \leq S C_{a}$ and $S C_{j} \leq S C_{b}$, compute

$$
H_{a}=H_{a}+\left(r_{b} \| r_{b}^{\prime}\right) X_{b}+\Sigma_{j_{\epsilon} J}\left(r_{j} \| r_{j}^{\prime}\right) X_{j}(\bmod N),
$$

where $\boldsymbol{J}=\left\{j \mid\left(S C_{j} \leq S C_{b}\right)\right.$ AND NOT $\left.\left(S C_{j} \leq S C_{a}\right)\right\}$.

Step 2: For all the security classes $S C_{i}$ that have the relationship $S C_{a} \leq S C_{i}$, do

$$
H_{i}=H_{i}+\Sigma_{j_{\epsilon} J}\left(r_{j} \| r_{i}^{\prime}\right)^{*} X_{j}(\bmod N),
$$

where $\boldsymbol{J}=\left\{j \mid\left(S C_{j} \leq S C_{a}\right)\right.$ AND NOT $\left(S C_{b} \leq S C_{i}\right)$ AND NOT $\left.\left(S C_{j} \leq S C_{i}\right)\right\}$.

Example 3: Suppose that a new relationship is added between $S C_{5}$ and $S C_{6}$ in Fig. 1 , and $S C_{6} \leq S C_{5}$; then the resulting hierarchy is shown in Fig. 4. $\left\{S C_{1}, S C_{2}, S C_{3}\right.$, $\left.S C_{5}\right\}$ all have the access right to $S C_{6}$; therefore, CA will compute:

$$
\begin{aligned}
& H_{5}=H_{5}+\left(r_{6} \| r_{6}^{\prime}\right)^{*} X_{6}(\bmod N) \\
& H_{2}=H_{6}+\left(r_{6} \| r_{6}^{\prime}\right)^{*} X_{6}(\bmod N)
\end{aligned}
$$

In the updated hierarchy, $S C_{5}$ can derive the secret key of $S C_{6}$. Since $S C_{5} \leq S C_{2}$ before the updating, $S C_{2}$ can have the access right to $S C_{6}$ after the relationship $S C_{6} \leq S C_{5}$ is added.

\subsection{Deleting a relationship}

Suppose that a relationship between $S C_{b}$ and $S C_{a}$ is deleted from a user hierarchy, then the relationship of $S C_{b} \leq S C_{a}$ will not exist. CA only recomputes the public parameters $H_{i}^{\prime} s$ for $S C_{b} \leq S C_{i}$, while other parameters remain unchanged. Before $\mathrm{CA}$ deletes the relationship of $S C_{b} \leq S C_{a}$, it will perform the following procedure:

Step 1: For $S C_{b} \leq S C_{a}$ and $S C_{j} \leq S C_{b}$, compute

$$
H_{a}=H_{a}-\left(r_{b} \| r_{b}^{\prime}\right) X_{b}-\Sigma_{j_{\epsilon} J}\left(r_{j} \| r_{j}^{\prime}\right) X_{j}(\bmod N),
$$

where $J=\left\{j \mid\left(S C_{j} \leq S C_{b}\right)\right.$ AND NOT $\left.\left(S C_{j} \leq S C_{a}\right)\right\}$.

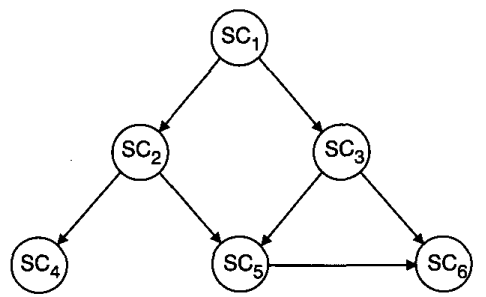

Fig. 4 The resulting hierarchy of adding the relationship $S C_{6} \leq S C_{5}$ IEE Proc-Computers \& Digital Techniques, Vol. 146, No. 5, September 1999 


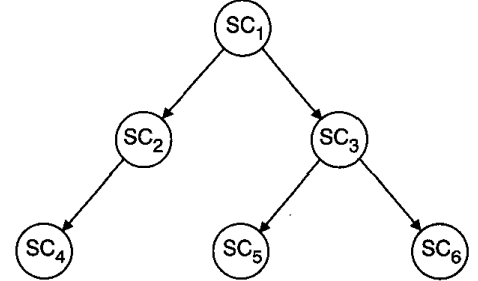

Fig. 5 The resulting hierarchy of deleting the relationship $S C_{5} \leq S C_{2}$

Step 2: For all the security classes $S C_{i}$ that have the relationship $S C_{a} \leq S C_{i}$, do

$$
H_{i}=H_{i}-\Sigma_{j_{\epsilon} J}\left(r_{j} \| r_{j}^{\prime}\right)^{*} X_{j}(\bmod N),
$$

where $J=\left\{j \mid\left(S C_{j} \leq S C_{a}\right)\right.$ AND NOT $\left(S C_{b} \leq S C_{i}\right)$ AND NOT $\left.\left(S C_{j} \leq S C_{i}\right)\right\}$.

Example 4: Suppose that the relationship $S C_{5} \leq S C_{2}$ in Fig. 1 is deleted, the resulting hierarchy is shown in Fig. 5. CA only needs to compute:

$$
H_{2}=H_{2}-\left(r_{5} \| r_{5}^{\prime}\right)^{*} X_{5}(\bmod N)
$$

and other parameters remain unchanged. $S C_{2}$ cannot derive the secret key of $S C_{5}$ in the updated hierarchy. It means that $S C_{2}$ no longer has the access authority to $S C_{5}$ 's information items.

\subsection{Changing a secret key}

When a user requests $\mathrm{CA}$ to change his secret key, CA must derive all the users' secret keys. In the security classes, we are able to change our secret keys freely in the issued system, and CA only needs to update the public information $C$ of the class. For example, if the key $S K_{i}$ of the security class $S C_{i}$ is changed to $S K_{i}^{\prime}$, the public information $C_{i}$ only needs to be updated as $C_{i}^{\prime}=\left(S K_{i}^{\prime} \| I D_{i}\right)$ $\left(\left(S K_{i}^{\prime} \| I D_{i}\right)+b_{i}\right) \bmod m_{i}$, and all other keys or information items need not be changed.

\section{Security analysis and discussions}

In this section, we present the security, the efficiency of our scheme, and the functional comparisons in sequence.

\subsection{Security}

As shown in the key derivation process, any security class with its secret information $H$ can derive his successors' keys easily; the opposite of this is not allowed because each security class only knows the public information $b, n$, and $C$ of its predecessor. For example, as shown in Fig. 1, if the $S C_{3}$ wants to use a single key to retrieve information from all his successors $S C_{5}$ and $S C_{6}, S C_{3}$ must firstly obtain $\mathrm{H}_{3}$. Since $\mathrm{H}_{3}$ consists of $\mathrm{H}_{5}$ and $\mathrm{H}_{6}$, which are made up of $\left(r_{5} \| r_{5}^{\prime}\right)$ and $\left(r_{6} \| r_{6}^{\prime}\right)$, respectively; $S C_{3}$ can thus derive $S K_{5}$ and $S K_{6}$ by using $\left(r_{5} \| r_{5}^{\prime}\right)$ and $\left(r_{6} \| r_{6}^{\prime}\right)$. Certainly, $S C_{3}$ is able to use a single key to retrieve information from all his successors $S C_{5}$ and $S C_{6}$.

If two or more users at a lower level in the system collaborate to derive a higher level key, from Step 5 of the key generation algorithm, we understand that all successors of some security class can only collaborate to obtain $H$ of the class by using all of their own $\left(r, r^{\prime}\right)$ and $X$. Even if they obtain their predecessor's $H$, it cannot provide them with enough information to reveal the secret key because they can only know what they own. Thus, only under the disclosure of its corresponding data $\left(r, r^{\prime}\right)$ can the predecessor's secret key $S K$ be revealed.

Through Dirichlet's theorem on primes, there are infinite integers that we can choose for $r$ and $r^{\prime}$ when $b \geq 1$. Hence, what we are concerned about is how to choose these two integers $r$ and $r^{\prime}$ in our scheme. Let $r$ and $r^{\prime}$ be integers of 10 digits, thus the entire exhaustive searching space needs $10^{20}$ digits for $m$. Suppose that there are $10^{11}$ digits being searched per day, the entire computation would take about $10^{9}$ days. Therefore, the security of our scheme is determined by the careful selection of $\left(r, r^{\prime}\right)$. Furthermore, a small choice for modulus $n$ is possible since this value is kept secret in this scheme unlike Rabin's public key scheme where the modulus is public.

\subsection{Efficiency}

Each security class needs to store the secret information $\left(r, r^{\prime}, X, H, S K\right)$, and the public information $(b, n, C)$. Compared with other schemes $[9-11,20]$, our scheme seems to need more storage for storing $b, r, r^{\prime}, X, n$, and $H$. However, the whole storage required for actual practice is small.

First, by the security classes or CA, $\left(r, r^{\prime}\right)$ and $b^{\prime}$ s values can be randomly selected. In order to reduce the values of the primes $(p, q)$, the values should be selected to be as small as possible. But, it may be argued that the primes $(p, q)$, generated by the small $\left(r, r^{\prime}\right)$ and $b$ of 10-digit, will make the scheme insecure because Rabin's scheme is based on the difficult computation of factoring the product of two primes of 100-digit [4]. However, from the key generation and derivation algorithms, we know that $m$ will not show up any more after generating the public information $C$. This means that our scheme is secure enough to avoid the attack of factoring $m$ by using $m$ as a 20-digit number. Therefore, the storage space of secret key $S K$ and the public information $C$, where $S K$ and $C$ are 20 digits, is much smaller than that needed in $[9-11,13,15,20]$.

Next, the storage of an equation $H$ is proportional to the number of the security class $S C$ 's successors. However, it is a time-space tradeoff in practice. We generate $H$ for the non-leaf security class so that the key derivation process will become more efficient, and the derivation path will be found by deriving the successors' key step by step. Let $k$ be the number of the security class $S C$ 's successors, then the storage needed is about $k *\lceil\log P\rceil$ bits for $H$. Therefore, the total storage required is also small.

Finally, in our key generation or derivation and the insertion of new security classes, both the computation of Rabin's public key cryptosystem and the construction of the equation $H$ are the key steps. In Rabin's system, encryption function is a second-order polynomial that computes the modulus of the product of two primes, and the decryption function is a square-root function that computes the modulus of one of the two primes. Thus, it is easy to see that the computational complexity of the key assignment dominated the cost of finding $y_{i}^{\prime} \mathrm{s}$ values in the equation $H$. Knuth [21] shows that the time complexity of the finding of $y_{i}$ is $O\left(\log ^{3} n_{i}\right)$.

\subsection{Functional comparisons}

In Table 1, we list the functions of our scheme compared with the previously proposed schemes. 
Table 1: Functional comparisons

\begin{tabular}{|c|c|c|c|c|}
\hline Comparison items & AT & HL & WH & PRO \\
\hline $\begin{array}{l}\text { Hierarchy type } \\
\text { Key deriving } \\
\text { method }\end{array}$ & $\begin{array}{l}\text { poset } \\
\text { direct }\end{array}$ & $\begin{array}{l}\text { poset } \\
\text { direct }\end{array}$ & $\begin{array}{l}\text { poset } \\
\text { direct }\end{array}$ & $\begin{array}{l}\text { poset } \\
\text { indirect }\end{array}$ \\
\hline $\begin{array}{l}\text { Key assignment } \\
\text { complexity }\end{array}$ & $\begin{array}{l}\text { exponential } \\
\text { operation }\end{array}$ & $\begin{array}{l}\text { exponiential } \\
\text { operation }\end{array}$ & $\begin{array}{l}\text { CRT + one-way } \\
\text { function }\end{array}$ & $\begin{array}{l}\text { CRT + one-way } \\
\text { function }\end{array}$ \\
\hline $\begin{array}{l}\text { Key deriving } \\
\text { complexity }\end{array}$ & $\begin{array}{l}\text { exponential } \\
\text { operation }\end{array}$ & $\begin{array}{l}\text { exponential } \\
\text { operation }\end{array}$ & $\begin{array}{l}\text { modular } \\
\text { operation }\end{array}$ & $\begin{array}{l}\text { modular } \\
\text { operation + } \\
\text { square root }\end{array}$ \\
\hline $\begin{array}{l}\text { Adding/deleting } \\
\text { classes }\end{array}$ & $\begin{array}{l}\text { updating entire } \\
\text { parameters }\end{array}$ & $\begin{array}{l}\text { updating entire } \\
\text { parameters }\end{array}$ & $\begin{array}{l}\text { updating entire } \\
\text { parameters }\end{array}$ & $\begin{array}{l}\text { updating partial } \\
\text { parameters }\end{array}$ \\
\hline $\begin{array}{l}\text { Adding/deleting } \\
\text { relätionship }\end{array}$ & $\begin{array}{l}\text { updating entire } \\
\text { parameters }\end{array}$ & $\begin{array}{l}\text { updating entire } \\
\text { parameters }\end{array}$ & $\begin{array}{l}\text { updating partial } \\
\text { parameters }\end{array}$ & $\begin{array}{l}\text { updating partial } \\
\text { parameters }\end{array}$ \\
\hline $\begin{array}{l}\text { Changing secret } \\
\text { key }\end{array}$ & $\begin{array}{l}\text { updating entire } \\
\text { parameters }\end{array}$ & $\begin{array}{l}\text { updating entire } \\
\text { parameters }\end{array}$ & $\begin{array}{l}\text { updating entire } \\
\text { parameters }\end{array}$ & $\begin{array}{l}\text { updating partial } \\
\text { parameters }\end{array}$ \\
\hline $\begin{array}{l}\text { Storage for public } \\
\text { informiation }\end{array}$ & large & large & large & fixed and small \\
\hline
\end{tabular}

Remark: AT: Akl and Taylor's scheme [9]; HL: Harn and Lin's scheme [11]; WH: Wu and He's scheme [17]; PRO: our proposed scheme.

\section{Conclusion}

For an ärbitrary poset user hierarchy, we have presented a dynamic access control scheme. It can ensure that the predecesssor's secret key will not be revealed by conspiracy on the part of any successors. In other words, our scheme hās been shown to be sècurè.

The proposed scheme contains the following three chäaractëristics:

(1) The key generation and derivation algorithms are simple : For the user, the successors' keys can be derived directly rather than stepwise.

(2) The updating of the existing keys can be avoided when a new class is added into the system or an old one is deleted from the system.

(3) Since any security class can select its own secret key for convenience and change its secret key for security reasons, the key selection becomes more flexible.

\section{Acknowledgments}

The authors woüld like to thank the anonymous referees for their constructive comments which improved the quality of this paper. This work was supported in part by the National Science Council under grant NSC 88 - 2516 $\mathrm{S} 131-003$

\section{Rëferénces}

1 DAVIDA, G.I., WELLES, D.L., and KAM, J.B.: 'A database encryption system with subkeys', ACM Trans. Database Syst., 1981,6, (2), pp. 312 328

2 DENNING, D.E.: Cryptography and Data Security, Chap. 2 (AddisonWesley, Massachusetts, 1983)

3 DENNING, D.E.; AKL, S.G., MORGENSTERN, M., and NEUMANN, P.G.: 'Views for multilevel databasè security', Proc., 1986 IEEE Symp. on Secirity and Privacy, 7-9, April 1986, Oakland, CA, pp. 156-172
4 McCULLOUGH, D.: 'Specifications for multilevel security and a hookup property', Proc. 1987 IEEE Symp. on Security and Privacy, 27-29, April 1987, Oakland, CA, pp. 161-166

5 McHUGH, J., and MOORE, A.P.: 'A security policy and formal top level specification for a multilevel secure local area network', Proc. 1986 IEEE Symp, on Security and Privacy, 7-9, April 1986, Oakland, CA, pp. $34-39$

6 FRAIM, L.J.: 'Scomp: a solution to multilevel security problem', IEEE Comput.; 1983, pp. 26-143

7 WILLIAMS, H.C.: 'A modification of the RSA public-key encryption procedure', IEEE Trans. Inf. Theory, 1980, IT-26, (6), pp. 729-729

8 QUISQUATER, J.J., and COUVREUR, C.: 'Fast decipherment algorithm for RSA public-key cryptosystem', Electron. Lett., 18, pp. 905907,1982

9 AKL, S.G., and TAYLOR, P.D.: 'Cryptographic solution to a problem of access control in a hierarchy', ACM Trans. Comput. Syst., 1983, 1, (3), pp. 239-247

10 MacKINNON, S.T., TAYLOR, P.D., MEUJER, H., and AKL, S.G.: 'An optimal algorithm for assigning cryptographic keys to control access in a hierarchy', IEEE Trans. Comput., 1985, C-34, (9), pp. 797-802

11 HARN, L., and LIN, H.Y.: 'A cryptographic key generation scheme for multilevel data security', Comput. Secur., 1990, 9, pp. 539-546

12 CHANG, C.C., and BUEHRER, D.J.: 'Access control in a hierarchy using a one-way trapdoor function', Comput. Math. with Appl., 1993, 26, (5), pp. 71-76

13 CHANG, C.C., HWANG, R.J., and WU, T.C.: 'Cryptographic key assignment scheme for access control in a hierarchy', Inf. Syst., 1992, 17, (3), pp. 243-247

14 CHICK G.C. and TAVARES, S.E. "Flexible access control with master keys', in 'Advances in Cryptology - CRYPTO'89, Springer-Verlag, $1990, \mathrm{pp} .316-322$

15 SANDHU, R.S.: 'Cryptographic implementation of a tree hierarchy for access control', Inf. Proc. Lett., 1988, 27, pp. 95-98

16 TSAI, H.M., and CHANG, C.C:: 'A cryptographic implementation for dynamic access control in a user hierarchy', Comput. Secur., 1995, 14, (2), pp. 857-959

17 WU, TC WU, TS, and HE, WH.: 'Dynamic access control scheme based on the Chinese remainder theorem' Comput. Syst. Sci. Eng. 1995, 10, (2), pp. 92-99

18 RABIN, M.O.: 'Digitized signatures and public-key function as intractable as factorization'. Technical Report, MIT/LCS/TR-212, MIT Lab. for Computer Science, 1979

19 RIBENBOIM, P.: 'The Book of Prime Number Records', 2nd edn., (Springer-Verlag, 1989)

20 LAIH, C.S., and HWANG, T.L.: 'A branch oriented key management solution to dynamic access control in a hierarchy", IEEE Trans. Softw. Eng., 1991, 17, (3), pp. 422-429

21 KNUTH, D.E.: 'The Art of Computer Programming, Seminumerical Algorithms', 2nd edn., (Addison-Wesley, Reading, MA, 1981) 